\title{
Secure Communication in Two-Way Relay Networks with Channel Estimation Errors
}

\author{
Yao Chenhong ${ }^{12 *}$, Pei Changxing ${ }^{1}$ and Guo Jing ${ }^{3}$ \\ ${ }^{1}$ State Key Laboratory of Integrated Services Networks, Xidian University, Xi'an, \\ 710071, Shaanxi Province, China \\ ${ }^{2}$ Xian University of Architecture and Technology, Xi'an 710055, Shaanxi \\ Province, China \\ ${ }^{3}$ Shanxi University of Science \& Technology Xi'an 710021, Shaanxi Province, \\ China \\ E-mail:xiaoyaoff@163.com
}

\begin{abstract}
In this paper, we investigate the secrecy outage probability (SOP) for the cooperative amplify-and-forward (AF) two-way relay networks (TWRN) with channel estimation (CE) errors under Rayleigh fading channels. In the proposed system, two sources simultaneously broadcast their respective information to all the relays firstly. Then, the optimal relay performs AF protocol on the received signals sent from the two sources and forward them to both sources. In both phases, An eavesdropper receives the signals from sources and relay nodes illegally. The performance of the proposed system is quantified by deriving the upper bound of the overall outage probability. Impact of CEs on the system is illustrated by simulations.
\end{abstract}

Keywords: Two-way relaying network, amplify-and-forward (AF), wiretap channel, channel estimation errors

\section{Introduction}

Due to their inherent broadcasting nature, wireless communication systems are prone to security threats. One possible way against eavesdropper is utilizing cryptographic encryption, eavesdroppers hardly recover the source information without the secret key. However, the encryption method requires considerable computational resources and communication overheads, which adds another layer of complexity in the design of networks [1]. Another new approach against eavesdropper is physical layer (PHY) security by exploiting the physical characteristics of wireless channels. This work was pioneered by Wyner, who introduced the wiretap channel in 1975 and established the possibility to transmit confidential messages without using secret keys [2]. In order to increase the secrecy rate of networks, cooperative relaying communication is introduced [3], the main objective is to boost the capacity of the primary links by decreasing simultaneously the capacity of the eavesdropper links. Meanwhile, there has been a growing of interest in two-way communications due to its bandwidth efficiency and potential applications to cellular and peer-to-peer networks [4].

One important feature for PHY security is that the channel state information (CSI), including which from the source to the destination as well as from the source to the eavesdropper, should be known by the source and/or the destination to enable signal processing such as beamforming and jamming. Most of the previous research about PHY secure wireless communication have assumed perfect CSI. However, in practical communication systems, CSI is observed by estimation algorithms and CE error is

* Corresponding Author 
inevitable. The reference [5] gives the optimal channel estimation and training design for TWRN and the corresponding problem with the assumption of time-selective channel is considered in [6]. In general, the CE error exists at all communication. Recently, physical layer security in the presence of imperfect $\mathrm{CE}$ attracts increasingly attention.

Current developments in PHY security are often based on the assumption of perfect CSI. In [7], authors investigate joint relay and jammer selection in two-way cooperative networks, consisting of two sources, one eavesdropper, and a number of intermediate nodes, with secrecy constraints. Specifically, the proposed schemes select two or three intermediate nodes to enhance security against the malicious eavesdropper. Some works are based on two-way relay networks with imperfect CSI. In [8], authors study the performance of bidirectional multi-relay cooperative networks in the presence of imperfect channel state information by means of the correlation coefficient of the estimated channel gains and their actual values, and present a power allocation scheme that minimizes the outage probability. In [9], authors investigate, the secrecy performance of a single-input single-output single antenna eavesdropper two-way relay wiretap channel, in which only statistical channel state information from the eavesdropper is available at the sources. They propose a secure adaptive transmission scheme for this two-way relay wiretap channel in which the sum effective secrecy throughput is utilized as the performance metric.

A number of analysis in the literature have considered performance in Two-Way Relay Networks With Channel Estimation Errors. In [10], The impact of channel estimation errors is investigated in two-way relay networks (TWRNs) with two different channel state information (CSI) estimation schemes, namely separate and cascaded, and the source nodes in the TWRN are assumed to have different estimation errors. In [11], the outage behavior of decode-and-forward (DF) relaying in the context of selective two-way cooperative systems is investigated. In[12], authors investigate the impact of outdated channel estimates on the overall performance of a two-way multi-relay system that employs a three-phase (3P) analog network coding (ANC)-based opportunistic relay selection (ORS) scheme under Rayleigh fading. In references [13]-[15], effects of imperfect CSI are studied in other wireless communications. In [13], authors analyze the impact of outdated CSI on the security performance in multi-input multi-output (MIMO) system, where the transmitter uses maximal-ratio transmission (MRT) and the receiver uses receive antenna selection (RAS). In their analysis, new closed-form expressions for the probability of the existence of a nonzero secrecy capacity and the secrecy outage probability are derived. In [14], authors analyze the effects of outdated CSI on the secrecy outage performance of MISO wiretap channels with transmit antenna selection. In [15], Yang W. et. al., investigate the secrecy outage probability for the cooperative decode-and-forward underlay cognitive radio networks (CRNs).

In [16], authors investigate jammer selection in a two-way decode-and-forward relay network with imperfect channel state information, under the assumption that the relay can decode received signals perfectly and when the jamming power is higher than that of source nodes. In [17], authors study the channel estimation and data detection problem for secure Amplify-and-Forward two-way relay networks, in which channel estimation errors of both the legal user and the eavesdropper are studied, and they derive the closedform expression of channel capacity with imperfect channel state information. In [18], joint relay/jammer selection and power control with friendly jammers for physical layer security in two-way relay networks are studied, in which the impact of channel estimation error on the wiretap channel is also considered. To our best knowledge, seldom works study the secrecy outage probability of AF TWRN when channel estimation errors exists.

Motivated by all of the above, we firstly build up a secure amplify-and-forward (AF) TWRN model with channel estimation between the sources and relay nodes. And then, the upper bound of the overall outage probability for the proposed system is investigated. Finally, simulation results indicates that estimation errors decrease the outage 
performance of the system both in the same CEs and different CEs for different sourcerelay channels.

\section{System Model}

Consider a two-way amplify-and-forward (AF) relaying system, where two sources $S_{1}$ and $S_{2}$ exchange information with the help of $K$ cooperating AF relay nodes $k, k \in\{1, \ldots$, $K\}$ in the presence of an eavesdropper $E$, as shown in Figure 1. All channels are assumed quasi-static, reciprocal, and subject to independent and non-identically distributed (i.ni.d.) flat Rayleigh fading. Furthermore, assume that data transmissions are performed using a time division duplex. As such, the channel coefficients are assumed to remain constant over a block and correlated across blocks, where the block duration depends on the channel coherence time, and every channel gain between the source and $\mathrm{k}$ can be modeled as a complex Gaussian random variable, that is, $h_{a, b} \square \mathcal{C N}\left(0, \Omega_{a b}\right)$, where $a, b \in\left\{S_{1}, S_{2}, k, E\right\}$, and $h_{a, b}=h_{b, a}$. We assume that all terminals are single-antenna devices and operate in a half-duplex mode, and there is no direct path between $S_{1}$ and $S_{2}$. We also assume here the additive white Gaussian noise (AWGN) at all nodes is independent and identically distributed (i.i.d.) $\mathrm{CN}\left(0, N_{0}\right)$.

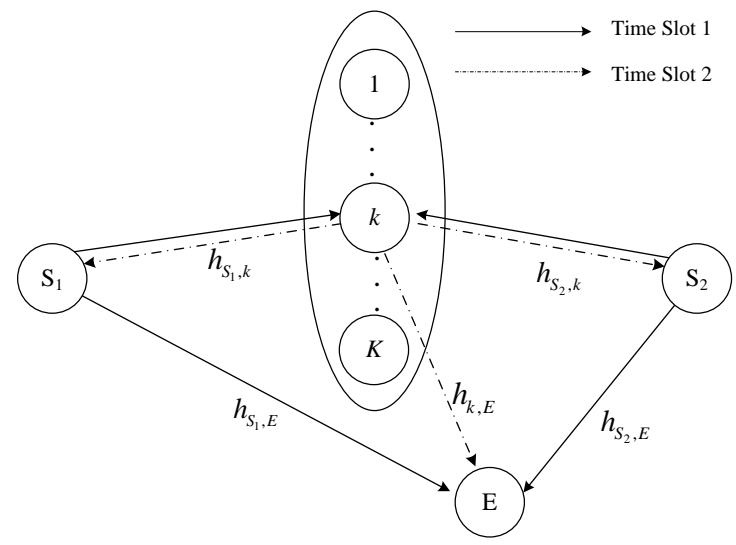

Figure 1. System Model of TWRN with an Eavesdropper

In the first time slot, $S_{1}$ and $S_{2}$ transmit their signals $x_{1}$ and $x_{2}$ to all the relays simultaneously. So the received signal at relay $k$ and the received signal at eavesdropping node can be expressed as:

$y_{k}=\sqrt{P_{S_{1}}} h_{S_{1}, k} x_{1}+\sqrt{P_{S_{2}}} h_{S_{2}, k} x_{2}+n_{k}$,

$y_{e_{1}}=\sqrt{P_{S_{1}}} h_{S_{1}, \mathrm{E}} x_{1}+\sqrt{P_{S_{2}}} h_{S_{2}, \mathrm{E}} x_{2}+n_{e_{1}}$

where $P_{S 1}$ and $P_{S 2}$ are the transmitting power of $S_{1}$ and $S_{2} . h_{S 1, k}$ and $h_{S 2, k}$ stand for the channel gains of $S_{1} \rightarrow k$ and $S_{2} \rightarrow k$, respectively. nk and ne1 are the AWGN at relay node $\mathrm{k}$ and the eavesdropper node $\mathrm{E}$ in the first time slot, respectively. We model the relation between the channel coefficient $h_{S_{i}, k}$ and its estimation $\hat{h}_{S_{i}, k}$ as:

$h_{S_{i}, k}=\hat{h}_{S_{i}, k}+e_{S_{i}, k}, i=1,2$

where $e_{S_{i}, k}$ is distributed as $C N\left(0, \sigma_{S_{i}, k}^{2}\right)$, thus, $\hat{h}_{S_{i}, k}$ is distributed as $C N\left(0, \Omega_{S_{i}, k}-\sigma_{S_{i}, k}^{2}\right)$. 
In the second time slot, the received signal at relay $k$ is amplified with the gain $G=\sqrt{P_{k} /\left(P_{S_{1}}\left|\hat{h}_{S_{1}, k}\right|^{2}+P_{S_{2}}\left|\hat{h}_{S_{2}, k}\right|^{2}+N_{0}\right)}$, and retransmitted to $S_{1}$ and $S_{2}$.

Since the sources know their own signal, i.e., S1 knows x1 and S2 knows x2, after the self-interference parts are subtracted [1], the received signals at the source $\mathrm{Si}$ and the eavesdropper are expressed as:

$$
\begin{gathered}
y_{S_{i}}=G h_{S_{i}, k} y_{k}+n_{S_{i}}-\sqrt{P_{S_{i}}} G\left|\hat{h}_{S_{i}, k}\right|^{2} x_{i} \\
=\underbrace{G \sqrt{P_{S_{j}}} \hat{h}_{S_{i}, k} \hat{h}_{S_{j}, k} x_{j}}_{\text {desired signal }}+\underbrace{G \sqrt{P_{S_{j}}} \hat{h}_{S_{i}, k} e_{S_{j}, k} x_{j}+G \sqrt{P_{S_{j}}} e_{S_{i}, k} \hat{h}_{S_{j}, k} x_{j}+G \sqrt{P_{S_{j}}} e_{S_{i}, k} e_{S_{j}, k} x_{j}}_{\text {imperfect-CSI noise }(1)} \\
+\underbrace{2 G \sqrt{P_{S_{i}}} \hat{h}_{S_{i}, k} e_{S_{i}, k} x_{i}+G \sqrt{P_{S_{i}}} e_{S_{i}, k}^{2} x_{i}}_{\text {imperfect-CSI noise(2) }}+\underbrace{G \hat{h}_{S_{i}, k} n_{k}+G e_{S_{S_{i}, k}} n_{k}+n_{S_{i}}}_{\text {white noise }}, i, j \in\{1,2\}, i \neq j \\
y_{e_{2}}=G h_{k, \mathrm{E}} y_{k}+n_{e_{2}} \\
=G \sqrt{P_{S_{i}}} h_{k, \mathrm{E}} \hat{h}_{S_{i}, k} x_{i}+G \sqrt{P_{S_{i}}} h_{k, \mathrm{E}} e_{S_{i}, k} x_{i}+G \sqrt{P_{S_{j}}} h_{k, \mathrm{E}} \hat{h}_{S_{j}, k} x_{j} \\
+G \sqrt{P_{S_{j}}} h_{k, \mathrm{E}} e_{S_{j}, k} x_{j}+G h_{k, \mathrm{E}} n_{k}+n_{e_{2}}, i, j \in\{1,2\}, i \neq j
\end{gathered}
$$

The received SNRs of link $S_{\mathrm{j}} \rightarrow k \rightarrow S_{\mathrm{i}} i, j \in\{1,2\}, i \neq j(\mathrm{i}, \mathrm{j})$ is given by

$$
\begin{aligned}
& \gamma_{i}=G^{2} P_{S_{j}} \hat{h}_{S_{i}, k} \hat{h}_{S_{j}, k} /\left(G^{2} P_{S_{j}}\left|\hat{h}_{S_{i}, k}\right|^{2} \sigma_{S_{j}, k}^{2}+G^{2} P_{S_{j}}\left|\hat{h}_{S_{j}, k}\right|^{2} \sigma_{S_{i}, k}^{2}+G^{2} P_{S_{j}} \sigma_{S_{i}, k}^{2} \sigma_{S_{j}, k}^{2}\right. \\
& \left.+4 G^{2} P_{S_{i}}\left|\hat{h}_{S_{i}, k}\right|^{2} \sigma_{S_{i}, k}^{2}+G^{2} P_{S_{i}} \sigma_{S_{i}, k}^{4}+G^{2}\left|\hat{h}_{S_{i}, k}\right|^{2} N_{0}+G^{2} \sigma_{S_{i}, k}^{2} N_{0}+N_{0}\right)
\end{aligned}
$$

$, i, j \in\{1,2\}, i \neq j$

Assume the statistic knowledge of the eavesdroppers link could be available from long term eavesdropper supervision in practice, then we can use the expectation $\mathrm{E}\left[\left|h_{k, \mathrm{E}}\right|^{2}\right]$ and $\mathrm{E}\left[\left|h_{S_{i}, \mathrm{E}}\right|^{2}\right]$ instead of $\left|h_{k, \mathrm{E}}\right|^{2}$ and $\left|h_{S_{i}, \mathrm{E}}\right|^{2}$, respectively.

For simplicity, consider the eavesdropper adopts the selection combing (SC) to combine the received signals within two time slots. Thus, the received SNRs of the eavesdropper for $\mathrm{xi}, i, j \in\{1,2\}, i \neq j(\mathrm{i}, \mathrm{j})$ is given by:

$$
\gamma_{E_{i}}=\max \left\{\frac{P_{S_{i}} E\left[\left|h_{S_{i}, \mathrm{E}}\right|^{2}\right]}{\sigma_{N_{S_{i}, e_{1}}}^{2}}, \frac{G^{2} P_{S_{i}}\left|\hat{h}_{S_{i}, k}\right|^{2} E\left[\left|h_{k, \mathrm{E}}\right|^{2}\right]}{\sigma_{N_{S_{i}, e_{2}}}^{2}}\right\} \text {, }
$$

where 


$$
\begin{aligned}
& \sigma_{N_{S_{i}, e_{1}}}^{2}=P_{S_{j}}\left|h_{S_{j}, E}\right|^{2}+N_{0}, \\
& \sigma_{N_{S_{i}, e_{2}}}^{2}=G^{2} P_{S_{i}}\left|h_{k, E}\right|^{2} \sigma_{S_{i}, k}^{2}+G^{2} P_{S_{j}}\left|h_{k, E}\right|^{2}\left|\hat{h}_{S_{j}, k}\right|^{2}+G^{2} P_{S_{j}}\left|h_{k, E}\right|^{2} \sigma_{S_{j}, k}^{2}+G^{2}\left|h_{k, E}\right|^{2} N_{0}+N_{0}
\end{aligned}
$$

The instantaneous capacity of the wiretap channel for xi and the instantaneous capacity between $\mathrm{Si}$ and the eavesdropper $\mathrm{E}$ can be expressed as:

$$
\begin{aligned}
& C_{i}=\frac{1}{2} \log _{2}\left(1+\gamma_{j}\right) \\
& C_{E_{i}}=\frac{1}{2} \log _{2}\left(1+\gamma_{E_{i}}\right)
\end{aligned}
$$

respectively. Thus, the secrecy rate for source $\mathrm{Si}$ is

$R_{s_{i}}=\left[\frac{1}{2} \log _{2}\left(1+\gamma_{j}\right)-\frac{1}{2} \log _{2}\left(1+\gamma_{E_{j}}\right)\right]^{+}, i, j=1,2 ; i \neq j$

where $[x]^{+} \square \max \{0, x\}$.

Since the overall performance of the considered system is governed by the performance of the weakest source. Taking into account the wiretap channel, the optimal relay selection criterion can be expressed as:

$$
k^{*}=\arg \max _{k \in\{1,2, \ldots, K\}}\left\{\min \left(\frac{1+\gamma_{1}}{1+\gamma_{E_{2}}}, \frac{1+\gamma_{2}}{1+\gamma_{E_{1}}}\right)\right\} \text {, }
$$

\section{Performance Analysis}

In this section, outage probability of the above-mentioned relay selection schemes under Rayleigh fading channel is analyzed, and expression for the overall outage probability is derived.

With the above selection scheduling, the overall outage probability is given by:

$$
\begin{gathered}
P_{\text {out }}=\operatorname{Pr}\left\{\max _{k \in\{1,2, \ldots, K\}}\left\{\min \left(\frac{1+\gamma_{1}}{1+\gamma_{E_{2}}}, \frac{1+\gamma_{2}}{1+\gamma_{E_{1}}}\right)\right\} \leq 2^{2 R}-1\right\} . \\
=\prod_{k=1}^{K} \operatorname{Pr}\left\{\min \left(\frac{1+\gamma_{1}}{1+\gamma_{E_{2}}}, \frac{1+\gamma_{2}}{1+\gamma_{E_{1}}}\right) \leq 2^{2 R}-1\right\} \\
\left.=\prod_{k=1}^{K}\left\{1-\operatorname{Pr}\left\{\min \left(\frac{1+\gamma_{1}}{1+\gamma_{E_{2}}}, \frac{1+\gamma_{2}}{1+\gamma_{E_{1}}}\right) \geq 2^{2 R}-1\right\}\right\}\right) \\
=\prod_{k=1}^{K}\left\{1-\operatorname{Pr}\left\{\frac{1+\gamma_{1}}{1+\gamma_{E_{2}}} \geq 2^{2 R}-1\right\} \operatorname{Pr}\left\{\frac{1+\gamma_{2}}{1+\gamma_{E_{1}}} \geq 2^{2 R}-1\right\}\right\}
\end{gathered}
$$


The two probability expression are parameter symmetrical, so we analysis the first one.

$$
\begin{aligned}
& \operatorname{Pr}\left\{\frac{1+\gamma_{1}}{1+\gamma_{E_{2}}} \geq \gamma_{t h}\right\} \leq \operatorname{Pr}\left\{\frac{1+\gamma_{1}}{\max \left\{\frac{P_{S_{2}}\left|h_{S_{2}, \mathrm{E}}\right|^{2}}{\sigma_{N_{S_{2}, e_{1}}}^{2}}, \frac{G^{2} P_{S_{2}}\left|\hat{h}_{S_{2}, k}\right|^{2}\left|h_{k, \mathrm{E}}\right|^{2}}{\sigma_{N_{S 2, e_{2}}}^{2}}\right\}} \geq \gamma_{t h}\right\} \\
& =\operatorname{Pr}\left\{\frac{1+\gamma_{1}}{\frac{P_{S_{2}}\left|h_{S_{2}, \mathrm{E}}\right|^{2}}{\sigma_{N_{S_{2}, e_{1}}}^{2}}} \geq \gamma_{\text {th }}\right\} \operatorname{Pr}\left\{\frac{1+\gamma_{1}}{\frac{G^{2} P_{S_{2}}\left|\hat{h}_{S_{2}, k}\right|^{2}\left|h_{k, \mathrm{E}}\right|^{2}}{\sigma_{N_{S 2, e_{2}}}^{2}}} \geq \gamma_{\text {th }}\right\} \\
& =P_{\text {out }}(1) P_{\text {out }}(2)
\end{aligned}
$$

Substituting (6) and (7) into (15), we can simplify $P_{\text {out }}(1)$ as:

$$
\begin{aligned}
& P_{\text {out }}(1)=\operatorname{Pr}\left\{a_{1}\left|\hat{h}_{S_{1}, k}\right|^{2}\left|\hat{h}_{S_{2}, k}\right|^{2}+a_{2} a_{5}\left|\hat{h}_{S_{1}, k}\right|^{2}+a_{3} a_{5}\left|\hat{h}_{S_{2}, k}\right|^{2}+a_{4} a_{5} \geq 0\right\}, \\
& \text { where } a_{1}=P_{k} P_{S_{2}}\left(P_{S_{1}} E\left[\left|h_{S_{1}, E}\right|^{2}\right]+N_{0}\right), \\
& a_{2}=\left(P_{k} P_{S_{2}} \sigma_{S_{2}, k}^{2}+4 P_{k} P_{S_{1}} \sigma_{S_{1}, k}^{2}+N_{0} P_{S_{1}}+P_{k} N_{0}\right), a_{3}=P_{k} P_{S_{2}} \sigma_{S_{1}, k}^{2}+N_{0} P_{S_{2}}, \\
& a_{4}=P_{k} P_{S_{2}} \sigma_{S_{1}, k}^{2} \sigma_{S_{2}, k}^{2}+P_{k} P_{S} \sigma_{S, k}^{4}+P_{k} \sigma_{S, k}^{2} N_{0}+N_{0}^{2}, a_{3}=P_{k} P_{S_{2}} \sigma_{S_{1}, k}^{2}+N_{0} P_{S_{2}}, \\
& a_{4}=P_{k} P_{S_{2}} \sigma_{S_{1}, k}^{2} \sigma_{S_{2}, k}^{2}+P_{k} P_{S_{1}} \sigma_{S_{1}, k}^{4}+P_{k} \sigma_{S, k}^{2} N_{0}+N_{0}^{2}, \\
& a_{5}=P_{S_{1}} E\left[\left|h_{S_{1}, E}\right|^{2}\right]+N_{0}-\gamma_{t h} P_{S_{2}} E\left[\left|h_{S_{2}, \mathrm{E}}\right|^{2}\right] .
\end{aligned}
$$

Set $\left|\hat{h}_{S_{1}, k}\right|^{2}=x,\left|\hat{h}_{S_{2}, k}\right|^{2}=y, A x y+B x+C y+D=z_{1} \cdot\left|\hat{h}_{S_{i}, k}\right|^{2}$ is an exponential distributed variable with parameter $\lambda_{i}=1 /\left[2\left(\Omega_{S_{i}, k}-\sigma_{S_{i}, k}^{2}\right)\right], i=1,2$. With the help of Eq. 3.324(1) and 3.471(9) in [19], we can obtain:

$$
\begin{aligned}
& P_{\text {out }}(1)=\operatorname{Pr}\left\{z_{1} \geq 0\right\} \\
& =e^{\frac{\lambda_{1} C+\lambda_{2} B}{A}}\left[\begin{array}{l}
-2 A \sqrt{\lambda_{1} \lambda_{2} F} K_{1}\left(2 \sqrt{\lambda_{1} \lambda_{2} F}\right)-2 A F \cdot K_{2}\left(2 \sqrt{\lambda_{1} \lambda_{2} F}\right) \\
+\frac{A}{\lambda_{2}}+2 E \sqrt{\lambda_{1} \lambda_{2} F} K_{1}\left(2 \sqrt{\lambda_{1} \lambda_{2} F}\right)-E
\end{array}\right]
\end{aligned}
$$


where $E=\frac{B C}{A}-D, F=\frac{B C}{A^{2}}-\frac{D}{A} . K_{v}(z)$ is Bessel function of imaginary argument.

Similarly, After substitution, the form of $P_{\text {out }}(2)$ is given by:

$$
\begin{aligned}
& P_{\text {out }}(2)=\operatorname{Pr}\left(z_{2} \geq 0\right) \\
& \quad=\operatorname{Pr}\left\{A_{2} x^{2} y+B_{2} y^{2} x+C_{2} x^{2}+D_{2} y^{2}+E_{2} x y+F_{2} x+G_{2} y+H \geq 0\right\} .
\end{aligned}
$$

The closed-form solve of (18) is hard to obtain. Specially, without regard to interferences between relay nodes, when the eavesdropper acts as an untrusted relay node, $\mathrm{E}\left[\left|h_{k, \mathrm{E}}\right|^{2}\right]=0$. Thus, $P_{\text {out }}(2)=1$. Then the upper bound of (15) can be derived.

\section{Numerical Results}

In this section, we present Monte-Carlo simulations to confirm impact of channel estimation errors on the overall outage probability. We assume that the average SNR in links from two sources to relay is equal, i.e., $P_{\mathrm{S} 1}=P_{\mathrm{S} 2}=P_{k}=1$, number of relay $K$ is 4 , The distance between two sources $S_{1}$ and $S_{2}$ is normalized to 1 . Without loss of generality, we set $\mathrm{d}_{1 k}=0.5$ for all the relays, and $\Omega_{S_{i}, k}=1, \mathrm{E}\left[\left|h_{k, \mathrm{E}}\right|^{2}\right]=\mathrm{E}\left[\left|h_{S_{i}, \mathrm{E}}\right|^{2}\right]=2$. The targeted transmission rate $\mathrm{R}=1 \mathrm{bit} / \mathrm{s} / \mathrm{Hz}$.

Figure 2 shows the actual simulation results for the overall outage probability when $K$ $=4$. The curve with $\sigma_{S_{1}, k}^{2}=\sigma_{S_{2}, k}^{2}=0$ represents the case of perfect channel estimation in the proposed system. As expected, we can see that either of the channel estimation errors $\sigma_{S_{1}, k}^{2}$ and $\sigma_{S_{2}, k}^{2}$ affects the overall outage probability, that is, as the CEs increase, whether they are different or not, the system outage performance degrades.

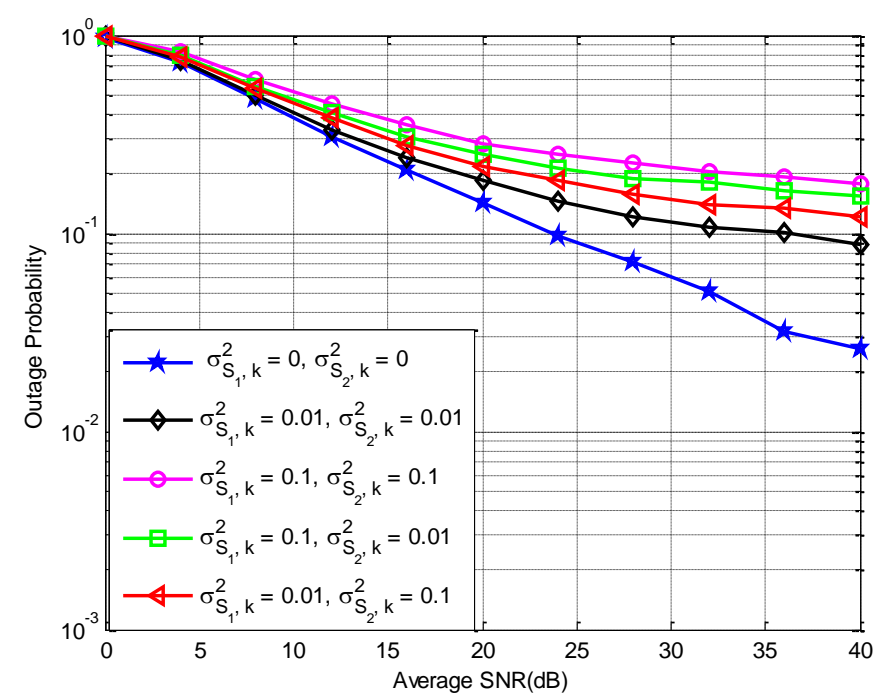

Figure 2. Outage Probability Against SNR for $K=4$ 


\section{Conclusion}

In this paper, we analysis the impact of the imperfect CSI (or CEs) in amplify-andforward two-way relay networks with an eavesdropper over independent flat Rayleigh fading channels. To research the performance of two way networks under security threat, relay selection is performed in accordance with the max-min rule and the expression of SOP is studied, and then Numerical results show that performance of the system degrades as either of the two CEs increases, no matter whether CEs are different or not.

\section{Acknowledgements}

The work was supported by the National Natural Science Foundation of China under Grant $(61072067,61372076)$, and funded by the State Key Laboratory of Integrated Services Networks under the Grant ISN 1001004, and by the National Science and Technology Major Project of China under Grant 2009ZX03007-003.

\section{References}

[1] Y. Liang, H. V. Poor and S. Shamai (Shitz), "Information theoretic security", Found. Trends Commun. Inf. Theory, vol. 5, nos. 4-5, (2008), pp. 355-580.

[2] A. D. Wyner, “The wire-tap channel”, Bell Syst. Tech. J., vol. 54, no. 8, (1975), pp. 1355-1387.

[3] L. Dong, Z. Han, A. P. Petropulu and H. V. Poor, "Secure wireless communications via cooperation", 46th Annual Allerton Conference on Communication, Control and Computing, Urbana-Champaign, IL, USA, (2008) Sep. 23-26, pp. 1132-1138.

[4] B. Rankov and A. Wittneben, "Spectral efficient protocols for half-duplex fading relay channels", IEEEJ. Select. Areas Commun., vol. 25, no. 2, (2007), pp. 379-389.

[5] F. Gao, R. Zhang and Y. C. Liang, "Optimal channel estimation and training design for two-way relay networks”, IEEE Trans. Commun., vol. 57, no. 10, (2009), pp. 3024-3033.

[6] G. Wang, F. Gao, W. Chen and C. Tellambura, "Channel estimation and training design for two-way relay networks in time-selective fading environments", IEEE Trans. Wireless Commun., vol. 10, no. 8, (2011), pp. 2681-2691.

[7] Chen J., Zhang R., Song L., Han Z. and Jiao B., "Joint Relay and Jammer Selection for Secure TwoWay Relay Networks", IEEE Transactions on Information Forensics \& Security, vol. 7, no. 1, (2011), pp. $1-5$.

[8] M. JafarTaghiyar, Sami Muhaidat, Jie Liang and MehrdadDianati, "Relay Selection with Imperfect CSI in Bidirectional Cooperative Networks", IEEE COMMUNICATIONS LETTERS vol. 16, no. 1, (2012), pp. 57-59.

[9] Liu C., Yang N., Yan S., Yuan J. and Malaney R., "Secure Adaptive Transmission in Two-way Relay Wiretap Channels", 2014 IEEE/CIC International Conference on Communications in China (ICCC), Shanghai, China, (2014) Oct 13-15, pp. 664 - 669.

[10] Pu J. W., Wang T. Y., Li S. H., Li C. P. and Li H. J., "Performance Analysis of Relay Selection in TwoWay Relay Networks With Channel Estimation Errors", IEEE Transactions on Broadcasting, vol. 61, no. 3, (2015), pp. 482-493.

[11] A. Hyadi, M. Benjillali and M.-S. Alouini, "Outage Performance of Decode-and-Forward in Two-Way Relaying with Outdated CSI”, IEEE Transactions on Vehicular Technology, vol. 64, no. 12, (2015), pp. 5940-5947.

[12] Yadav S. and Upadhyay P., "Impact of Outdated Channel Estimates on Opportunistic Two-Way ANCBased Relaying with Three-Phase Transmissions", IEEE Transactions on Vehicular Technology, vol. 6, no. 12, (2015), pp. 1-17.

[13] Hu J., Cai Y., Yang W. and Xu S., "Secrecy performance of MRT/RAS system with outdated CSI in MIMO wiretap channels", International Conference on Cyberspace Technology, (2013) Nov 23-23, pp. $422-427$.

[14] N. S. Ferdinand, D. B. da Costa and M. Latva-aho, "Effects of Outdated CSI on the Secrecy Performance of MISO Wiretap Channels with Transmit Antenna Selection", IEEE Communications Letters, vol. 17, no. 5, (2013), pp. 864-867.

[15] Yang W., Xu X., Cai Y. and Zheng B., "Secrecy outage analysis for cooperative DF underlay CRNs with outdated CSI", IEEE Wireless Communications and Networking Conference (WCNC), Istanbul, Istanbul, (2014) April 6-9, pp. 416 - 421.

[16] Wang J., Chen J., Duan H., Ba B. and Wu J., "Jammer selection for secure two-way DF relay communications with imperfect CSI", 16th International Conference on Advanced Communication Technology, Pyeongchang, Korea, (2014) Feb 16-19, pp. 300-303. 
[17] Liu Y., Zhong Z., Wang G. and Xu R., "Capacity Analysis for Secure Amplify-and-Forward Two-Way Relay Networks with Channel Estimation Errors", 5th IET International Conference on Wireless, Mobile and Multimedia Networks, Beijing, China, (2013) Nov 22-25, pp. 136-140.

[18] Jiang F., Zhu C., Peng J., W. Liu, Z. Zhu and Y. He, "Joint Relay and Jammer Selection and Power Control for physical Layer Security in Two-Way Relay Networks with Imperfect CSI", Wireless Personal Communications, vol. 85, no. 3, (2015), pp. 841-862.

[19] I. S. Gradshteyn and I. M. Ryzhik, "Table of Integrals", Series and Products, 6th edition. Academic Press, New York, (2000).

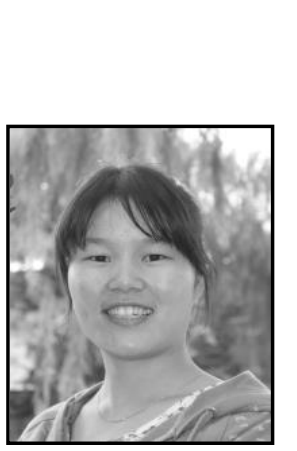

\begin{abstract}
Author
Yao Chenhong, She is currently working toward the Ph.D. degree in communication and signal processing with the State Key Laboratory of Integrated Services Networks, Xidian University, Xi'an, China. Her research interests include network measurement, wireless networks, relay networks, and parameter estimation theory. Email: xiaoyaoff@163.com.
\end{abstract}

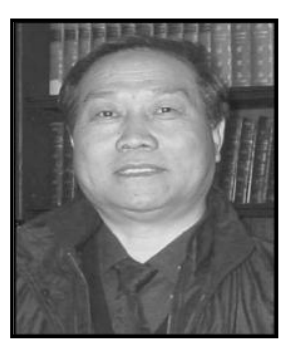

Pei Changxing, Professor, advisor for doctoral students, senior member of the China Institute of Communication, senior member of China Institute of Electronic, vice-director of the Electronics Institute of Shaanxi Province and the director of Working committee for Academic Exchange. He is mainly engaged in the fields of Radio communication, Anti-jamming communication, Network Measurement, Television system, High-frequency electronic circuits and Modern filter network. He holds two patents and applied for two. $\mathrm{He}$ also has been awarded the prize of scientific and technical accomplishment three times. He has authored eleven technical books. Over hundred papers were published in key journals and academic conferences.

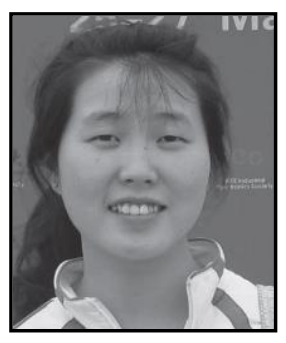

Guo Jing, Received the Ph.D. degree in communication engineering from Xidian University, Xi'an, China. She is currently a lecturer of the institute of Electrical \& Information Engineering, Shaanxi University of Science \& Technology, Xian, Shaanxi, China. Her current research interests include MIMO systems, relay-assisted and cooperative communications, and wireless communication theory. 
International Journal of Security and Its Applications

Vol. 10, No. 8 (2016) 